\title{
Evaluation of Schistosoma japonicum thioredoxin peroxidase-1 as a potential circulating antigen target for the diagnosis of Asian schistosomiasis
}

\author{
Adrian Miki C. MACALANDA ${ }^{1)}$, Jose Ma. M. ANGELES ${ }^{1}$, Kharleezelle J. MOENDEG $^{1}$, \\ Anh TM. DANG ${ }^{1}$ ), Luna HIGUCHI ${ }^{1)}$, Noboru INOUE ${ }^{1}$, , Xuenan XUAN ${ }^{1)}$, \\ Masashi KIRINOKI'), Yuichi CHIGUSA ${ }^{2)}$, Lydia R. LEONARDO3), \\ Elena A. VILLACORTE ${ }^{3)}$, Pilarita T. RIVERA ${ }^{3)}$, Yasuyuki GOTO ${ }^{4)}$ and \\ Shin-ichiro KAWAZU1)* \\ 1)National Research Center for Protozoan Diseases, Obihiro University of Agriculture and Veterinary Medicine, \\ Obihiro, Hokkaido 080-8555, Japan \\ ${ }^{2)}$ Department of Tropical Medicine and Parasitology, Dokkyo Medical University, Mibu, Tochigi 321-0293, Japan \\ ${ }^{3)}$ Department of Parasitology, College of Public Health, University of the Philippines Manila, Philippines \\ ${ }^{4)}$ Graduate School of Agricultural and Life Sciences, The University of Tokyo, Tokyo 113-8657, Japan
}

\section{J. Vet. Med. Sci.}

80(1): 156-163, 2018

doi: 10.1292/jvms.17-0579

Received: 29 October 2017

Accepted: 17 November 2017 Published online in J-STAGE: 28 November 2017
ABSTRACT. Asian schistosomiasis caused by Schistosoma japonicum is a serious zoonotic disease endemic in China, the Philippines and parts of Indonesia. Mass drug administration in endemic areas resulted to decline in disease severity and intensity. The low intensity of infection limits the use of current parasitological methods for schistosomiasis diagnosis. Detection of parasite circulating antigens might provide more informative result as it may indicate the true status of infection. In this study, S. japonicum thioredoxin peroxidase-1 (SjTPx-1) a 22 kDa secreted antioxidant enzyme expressed throughout the life stages of the parasite was evaluated for its potential use as a biomarker for schistosomiasis japonica infection. Rabbit polyclonal antibody and mouse monoclonal antibodies (mAbs) were raised against the recombinant SjTPx-1 (rSjTPx-1). The antibodies produced against the recombinant antigen was confirmed to detect the native SjTPx-1 in crude adult worm lysate. Likewise, the specific binding of mAbs to parasite TPx-1 and not to mammalian peroxiredoxin-1 orthologues was also confirmed. The double antibody sandwich ELISA developed in this study was able to detect at least $1 \mathrm{ng} / \mathrm{m} /$ of rSjTPx-1. In addition, this method was able to detect the antigen from all serum samples of experimentally infected rabbit and mice. The diagnostic potential of SjTPX-1 in human clinical samples was also evaluated, in which 4 out of 10 stool-confirmed serum samples had detectable levels of the antigen. The results suggest that SjTPx-1 can be a potential biomarker for Asian zoonotic schistosomiasis. KEY WORDS: Sandwich ELISA, Schistosoma japonicum, schistosomiasis biomarker, thioredoxin peroxidase-1

Schistosomiasis is a neglected tropical disease reported in 78 countries worldwide with at least 218 million people that require preventive chemotherapy [38]. The Asian zoonotic schistosomiasis caused by Schistosoma japonicum remains to be a threat to public health up to this date in China, the Philippines and parts of Indonesia. Several decades employing numerous control measures that include mass drug administration (MDA) with praziquantel resulted to the decrease in prevalence in the endemic areas $[18,29,31]$. Stool microscopy remains to be the only confirmatory test for schistosomiasis japonica. Although highly specific this test is labor intensive and requires a trained personnel and in recent times suffer decreased sensitivity due to the decrease in intensity of infection [19].

Mass screening of individuals by antibody-based serological assay has been shown to have better sensitivity as compared to traditional stool examination making it a promising alternative for schistosomiasis diagnosis [1]. However, antibodies are known to persist for several years even after cure [12]. This test therefore may not be able to differentiate past and present infection [12]. On the contrary, an antigen-based diagnosis can definitely detect present infection. Since circulating antigens are released by living

*Correspondance to: Kawazu, S.: skawazu@obihiro.ac.jp

O2018 The Japanese Society of Veterinary Science

This is an open-access article distributed under the terms of the Creative Commons Attribution Non-Commercial No Derivatives (by-nc-nd) License. (CC-BY-NC-ND 4.0: https://creativecommons.org/licenses/by-nc-nd/4.o/) 
parasites, the decrease in the number of adult worms upon treatment would result to a decrease in antigen levels in the blood of the host indicating response to drug therapy [12].

At present, a circulating cathodic antigen (CCA) point of care test (POCT) has been developed based on the CCA of $S$. mansoni [34]. This test has been successfully applied in numerous endemic areas in Africa [24]. Conversely, this does not remain true in Asian schistosomiasis infection where the POCT has been evaluated in a proof-of-concept study [31]. The current need for a specific antigen-based serodiagnosis has led to the evaluation of several antigen targets from the excretory and secretory products of the parasite that was shown to be potential biomarkers for schistosomiasis diagnosis $[11,28,30]$.

In this study, we assessed the applicability of S. japonicum thioredoxin peroxidase-1 (SjTPx-1) as a biomarker for Asian zoonotic schistosomiasis. SjTPx-1 has multiple biological functions; mainly as a key enzyme that combats reactive oxygen species [15]. Immunohistochemistry demonstrated the presence of SjTPx-1 in all life stages of S. japonicum as well as its extensive distribution on the surface tegument of the parasite and the tissues surrounding the egg in the liver [15, 21]. Additionally, this enzyme was recently identified as a component of the excretory and secretory products of the adult worm [11, 20]. Thus making SjTPX-1 a promising candidate for an antigen-based diagnosis.

Our previous studies on the use of SjTPX-1 as a biomarker for an antibody based ELISA showed good diagnostic capability in humans and water buffaloes [3, 4]. Although the ELISA OD values declined into negative levels after treatment (unpublished data), detection of both SjTPx-1 as antigen and its specific antibody may help to confirm the active stage of infection in the patients and the parasite-infected animals. Therefore in this study, we evaluated the potential of using SjTPX-1 as a target antigen for the diagnosis of Asian zoonotic schistosomiasis. Here we used a double antibody sandwich enzyme-linked immunosorbent assay (DAS-ELISA) to detect the presence of this antigen in the serum sample of experimentally infected animals and stool-confirmed patients from an endemic area in the Philippines.

\section{MATERIALS AND METHODS}

\section{Ethics statement}

Collection of serum samples from the patients in Sorsogon, the Philippines were carried out in compliance with the ethical clearance sought from the University of the Philippines-Manila (clearance no: UPM REB 2011-098). The animal experiments in this study were carried out in compliance with the guidelines for animal experimentation at Dokkyo Medical University and Obihiro University of Agriculture and Veterinary Medicine (permission numbers: 0006 and 28-30).

\section{Serum samples}

The serum from a Japanese white rabbit Icl strain (Ichikawa-ya, Chiba, Japan) percutaneously infected with 2,000 cercariae was collected at 11 weeks after the cercariae challenge. As well as sera from ten experimentally infected ICR mice Jcl strain (Clea Inc., Tokyo, Japan) percutaneously infected with 40 cercariae were collected at 8 weeks after the cercariae challenge. A total of 417 adult worm pairs were recovered from the infected rabbit and 2-6 adult worm pairs (average $=5$ ) from the infected mice. For the human clinical cases, serum samples from 10 individuals were collected when the stool samples were collected for microscopic examination of $S$. japonicum infection. These samples were collected from Sorsogon, the Philippines. For the negative control, serum samples from 7 non-endemic US volunteers were used.

\section{Cloning, expression and purification of recombinant SjTPx-1}

Cloning, expression and purification of recombinant SjTPx-1 (rSjTPx-1) were carried out as previously described with some modifications [4]. Briefly, first strand synthesis of complementary DNA (cDNA) was done using PrimeScript ${ }^{\mathrm{TM}}\left(\mathrm{Takara}^{\mathrm{Bio}}\right.$. Inc., Otsu). Forward primer 5'-GC GGA TCC ATG GTA CTG ATT CCA AAT-3' and reverse primer: 5'-GC CTC GAG TAA TCA GTG ATT CAC TTT-3' with BamHI/XhoI as the restriction sites (underlined) were used for polymerase chain reaction (PCR) amplification of the double strand DNA. The DNA fragment was cloned into pET-28a (+) vector (Novagen, Madison, WI, U.S.A.) for expression of the recombinant protein with 6xHis-tag on the $\mathrm{N}$ terminal in Escherichia coli (BL21) cells (Takara Bio Inc.). rSjPTx-1 was purified with affinity chromatography nickel nitrilotriacetic acid (Ni-NTA) protein purification kit (Qiagen, Hilden, Germany) and dialyzed against cold phosphate buffered saline (PBS) (pH 7.4) prior to use. The recombinant protein was quantified by Pierce ${ }^{\mathrm{TM}}$ BCA protein assay kit (Thermo Fisher Scientific, Rockford, IL, U.S.A.). Purity of rSjTPx-1 was assessed by sodium dodecyl sulfate polyacrylamide gel electrophoresis (SDS-PAGE).

\section{Cloning, expression and purification of recombinant mouse Prx-1}

Total RNA was extracted from a $50 \mathrm{mg}$ mouse liver tissue by homogenizing in $1 \mathrm{~m} l$ TRIzol ${ }^{\circledR}$ reagent (Life Technologies, Inc., Frederic, MD, U.S.A.). The complete coding sequence of mouse Prx-1 (MmPrx-1) which is the mouse orthologue for SjTPx-1 was PCR amplified from the cDNA synthesized using PrimeScript ${ }^{\mathrm{TM}}$ as previously described [16]. The forward primer 5'-GC $\underline{\mathrm{GGA}}$ TCC ATG TCT TCA GGA AAT GC-3' and reverse primer 5'-GC AAG CTT TCA CTT CTG CTT AGA GAA AT-3' with BamHI/ Hind III as their restriction sites (underlined) were used for the double strand DNA amplification. The PCR product was cloned into a pET-28a (+) vector. Prior to the transformation of $E$. coli (BL21) cells for the recombinant protein expression, sequencing was performed to confirm its identity with the database-deposited sequence. Recombinant MmPrx-1 (rMmPrx-1) was expressed and purified as previously described [4]. Protein concentration was measured by Pierce ${ }^{\mathrm{TM}}$ BCA protein assay kit while purity of rMmPrx-1 was assessed by SDS-PAGE. 


\section{Production of capture and detection antibodies}

Hyperimmune rabbit sera against rSjTPx-1 was produced by Wako pure chemicals (Wako Inc., Osaka, Japan). Briefly, 300 mg of rSjTPx-1 was injected subcutaneously into an 8-week old Japanese white rabbit. Booster injections were given twice at two weeks intervals each and finally, at 12 weeks post immunization the total serum was collected. IgG from the rabbit serum was purified by affinity chromatography using a MAbTrap ${ }^{\mathrm{TM}}$ antibody purification kit (GE Healthcare Bio-Sciences KK, Tokyo, Japan). Eluted IgG was desalted by PD-10 sepharose column (GE Healthcare) using PBS (pH 7.4). Concentration of the IgG (polyclonal antibody: pAb) was spectrophotometrically measured at $280 \mathrm{~nm}$ by NanoDrop 2000® (Thermo Fisher Scientific) whereas purity of the pAb was assessed by SDS-PAGE.

For the production of monoclonal detection antibodies an 8-week old female BALB/c mouse was immunized with $100 \mu \mathrm{g}$ rSjTPx-1 with the adjuvant TiterMax Gold® (Sigma-Aldrich, St. Louis, MO, U.S.A.). Two booster subcutaneous injections were given at two weeks interval each. The mouse was finally boosted with $50 \mu \mathrm{g}$ of antigen in PBS intravenously three days prior to harvesting the spleen. Splenocytes were fused with SP2/0 myeloma cells and selection was done in methylcellulose semi-solid media as previously described [7, 13]. Hybridomas secreting monoclonal antibodies (mAbs) were screened for their reactivity against rSjTPx-1 by indirect ELISA and further confirmed by western blotting. Isotype of mAbs were determined by IsoStrip ${ }^{\mathrm{TM}}$ mouse monoclonal antibody isotyping kit (Roche Diagnostics, Mannheim, Germany). Three hybridoma clones were selected for their stability and antibody secretion for expansion. IgG from culture supernatant was purified by MAbTrap ${ }^{\mathrm{TM}}$ purification kit and desalted in PD-10 desalting column. Concentrations of the IgGs (mAbs) were determined by NanoDrop 2000® spectrophotometer at $280 \mathrm{~nm}$ and their purity were evaluated by SDS-PAGE.

\section{Western blotting}

The specificity of mAbs against SjTpx-1 was determined by reacting them with rSjTPx-1, rMmPrx-1 and recombinant human Prx-1 (rHsPrx-1) (purchased from Sino Biological Inc., Beijing, China) in Western blotting as previously described with some modifications [5]. Briefly, $200 \mathrm{ng}$ of the recombinant proteins were resolved by SDS-PAGE and the polypeptides were transferred onto a polyvinylidene difluoride (PVDF) membrane. The membrane was incubated with the mAbs and the antigen-antibody reactions were detected by secondary antibody against mouse IgG conjugated with horseradish peroxidase (HRP) (GE Healthcare). Enzymatic activity was visualized by chemiluminescence using SuperSignal ${ }^{\mathrm{TM}}$ (Thermo Fisher Scientific) and a chemiluminescent detection machine ImageQuant LAS 500 (GE Electric). The reactivity of the antibodies against native SjTPx-1 was also evaluated with Western blotting. Adult worm lysate was prepared from 15 S. japonicum adult worms as previously described [5] and resolved by SDS-PAGE. The polypeptides were transferred onto a PVDF membrane and reacted with rabbit $\mathrm{pAb}$ and mouse mAb in a separate reaction chamber. The antigen-antibody reaction was detected either by HRP-conjugated anti-rabbit IgG or anti-mouse $\operatorname{IgG}$ antibody. Enzymatic activity was visualized by chemiluminescence as described earlier.

\section{DAS-ELISA}

Optimal concentrations of capture antibody and detection antibody were determined by checkerboard titration. Each well of 96well polystyrene plate (Thermo Fisher Scientific) was coated with $100 \mu l$ of 8, 4, 2 and $1 \mu \mathrm{g} / \mathrm{m} l$ of rabbit pAb as capture antibody in carbonate/bicarbonate buffer ( $\mathrm{pH} 9.6$ ) overnight at $4^{\circ} \mathrm{C}$. The wells were washed three times with PBS to remove unbound antibodies. The wells were then blocked by $5 \%$ skim milk in PBS at $37^{\circ} \mathrm{C}$ for $1 \mathrm{hr}$ and washed three times with PBS. Then, $100 \mu \mathrm{l}$ of rSjTPx-1 diluted in PBS at a concentration of $2 \mu \mathrm{g} / \mathrm{m} l$ was delivered into the wells as the positive control while PBS was used as a negative control. The wells were incubated for $1.5 \mathrm{hr}$ at $37^{\circ} \mathrm{C}$ then washed four times with PBS. One hundred $\mu l$ of detection antibody (anti-rSjTPx 1 mouse $\mathrm{mAb}$ ) was applied to each well at a concentration of 8, 4, 2 and $1 \mu \mathrm{g} / \mathrm{m} l$, respectively. The wells were incubated for $2 \mathrm{hr}$ at $37^{\circ} \mathrm{C}$ and washed for removing unbound antibodies. Then, $100 \mu l$ of HRP conjugated anti-mouse IgG antibody at a dilution of 1:10,000 was added to the wells. The wells were incubated for $1 \mathrm{hr}$ at $37^{\circ} \mathrm{C}$ then washed three times to remove any unbound antibodies. For the enzymatic reaction $100 \mu l$ of tetramethylbenzidine (TMB) (KPL Inc., Gaithesburg, MD, U.S.A.) was added to the wells and the reaction was allowed to proceed for $8 \mathrm{~min}$. The reaction was stopped by adding $50 \mu l$ of $1 \mathrm{M}$ phosphoric acid to the wells. The absorbance was measured at $450 \mathrm{~nm}$ with a microplate reader (Corona electric, Ibaraki, Japan). The optimal concentrations of capture $\mathrm{pAb}$ and detection $\mathrm{mAb}$ were determined as the combinations that give the highest positive to negative ratio. Following the optimization of antibody concentrations, $\mathrm{rSjTPx}-1$ antigen in different concentrations were tested with DAS-ELISA in order to determine the least amount of antigen that could be detected. The detection limit for SjTPx-1 by the DAS-ELISA was determined with the recombinant protein at $10^{5}, 10^{4}, 10^{3}, 10^{2}, 10^{1}, 10^{0}, 10^{-1}, 10^{-2}$ and $10^{-3} \mathrm{ng} / \mathrm{m} l \mathrm{in}$ PBS. Serum samples collected from experimentally infected animals and stool-confirmed patients were tested with the DAS-ELISA. Cut-off value was computed by taking the mean OD reading of negative controls plus 2 times the standard deviation of the mean OD readings. Prior to DAS-ELISA application, all serum samples were pretreated with trichloroacetic acid (TCA) as previously described [8].

\section{Statistical analysis}

Statistical analysis using student's $t$-test was performed using GraphPad Prism v 5.00 (GraphPad Software Inc., La Jolla, CA, U.S.A.). 
A

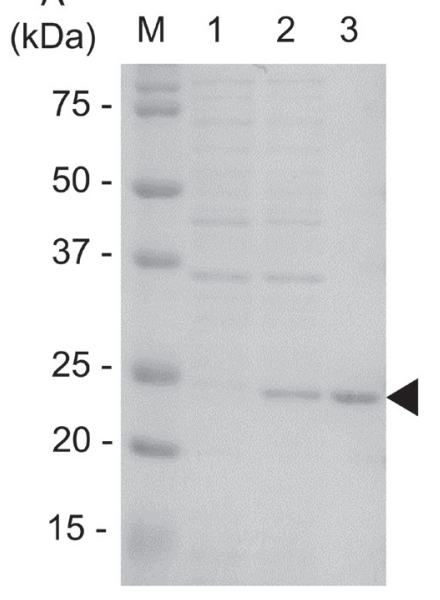

$\mathrm{B}$

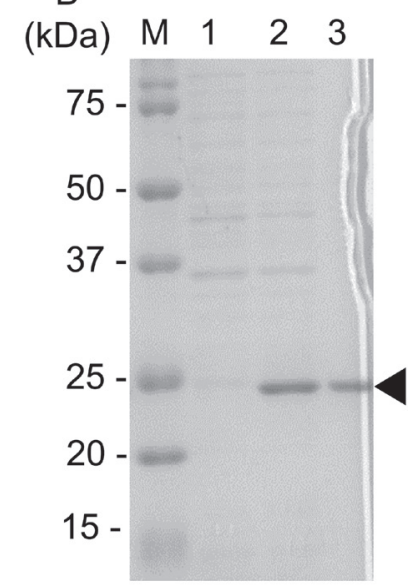

Fig. 1. Expression and purification of $\mathrm{rSjTPx}_{-1}(\mathrm{~A})$ and $\mathrm{rMmPrx}-1$ (B) The bands corresponding to the proteins were indicated with arrows ( $\mathbf{\Delta}$ ). Lane M - Molecular weight marker, Lane 1 - cell lysate prior to IPTG induction. Lane 2 - cell lysate after IPTG induction and Lane 3 - purified recombinant protein.

\section{RESULTS}

Cloning, expression and purification of recombinant SjTPX-1 and MmPrx-1 in E. coli

The complete coding sequence of SjTPx-1 and MmPrx-1 was amplified by PCR from the cDNA prepared from the adult worm and mouse liver respectively. SjTPx-1 complete coding sequence was $\sim 555$ bp in size while the coding sequence for MmPrx- 1 was $\sim 600 \mathrm{bp}$ (data not shown). After TA cloning and sequencing, they were confirmed for their identity with the database-deposited sequences (Sjp_0095720 and AAH86648.1). rSjTPx-1 and rMmPrx-1 were expressed in E.coli BL21 strain and were purified (Fig. $1 \mathrm{~A}$ and 1B). The analysis between the amino acid (AA) sequence of SjTPx-1 and those of mammalian orthologous Prx-1 showed considerable identity. AA sequence of S. japonicum TPx-1 shared $61.95 \%$ and $63.58 \%$ identity with those of mouse Prx-1 and human Prx-1, respectively (Supplementary Fig. S1).

\section{Production of capture and detection antibodies}

Rabbit pAb and mouse mAbs against rSjTPx-1 were produced. For mAbs, a total of seven hybridoma clones were developed and they were identified for $\operatorname{IgG}$ sub types (Supplementary Table S1). Based on the stability and antibody production ability, three clones, namely: A1F7, A1F11 and A1G7 were chosen. Among these clones, A1F7 was further selected to be used as detection mAb in DAS-ELISA because of its high secretion of $\mathrm{mAb}$ (data not shown). The antibodies were assessed by SDS-PAGE for their purity (data not shown).

\section{Specificity of the antibodies}

Equal amounts of rSjTPx-1, rMmPrx-1 and rHsPrx-1 were resolved by SDS-PAGE and transferred onto PVDF membrane for Western blotting. The equal loading of samples was confirmed by anti 6x-his tag mAb (Fig. 2A) the bands seen at $50 \mathrm{kDa}$ are the dimeric form typical of a 2-Cys Prx-1. The specificities of the three mAbs against rSjTPx-1 were verified (Fig. 2B-D). Likewise the reactivity of mAb A1F7 and pAb against the native SjTPx-1 were also confirmed (Fig. 2E).

\section{Optimization and evaluation of DAS-ELISA for SjTPx-1}

The optimal concentrations of capture pAb and detection mAb were determined by checkerboard titration (Supplementary Table $\mathrm{S} 2)$. Eight $\mu \mathrm{g} / \mathrm{m} l \mathrm{pAb}$ and $1 \mu \mathrm{g} / \mathrm{m} l \mathrm{mAb}$ were identified as the optimal concentrations of $100 \mu l$ antibody solutions per well for the DAS-ELISA. The lowest concentration of $\mathrm{rSjTPx}-1$ that could be detected by this combination was $1 \mathrm{ng} / \mathrm{ml}$ (Fig. 3A). The OD value recorded in the DAS-ELISA for the experimentally infected rabbit serum was significantly higher $(P \leq 0.001)$ than that of the normal rabbit serum (Fig. 3B). Likewise, the OD values recorded for the serum samples from the experimentally infected mice were significantly higher $(P \leq 0.001)$ than those of normal mice samples (Fig. $3 \mathrm{C})$. Testing of the stool microscopy positive individuals revealed that DAS-ELISA could detect circulating SjTPx-1 in 4 out of 10 serum samples (Fig. 3D).

\section{DISCUSSION}

In this study, we investigated the potential use of SjTPx-1 as an antigen marker for Asian schistosomiasis infection. In Schistosoma sp., several excretory and secretory antigens have been evaluated as potential biomarkers for infection [6, 11, 28, 32]. Among which, the detection of CCA in S. mansoni has been successfully applied as a field-friendly POCT [34]. The advantage of 
A

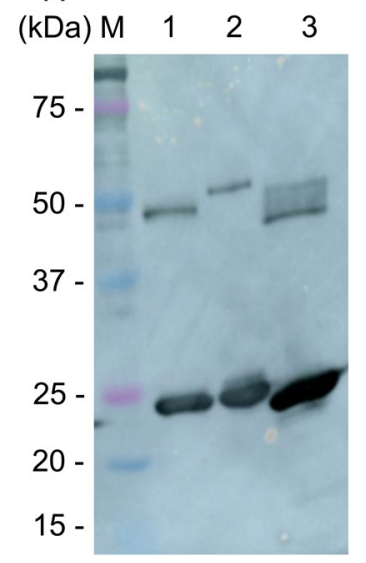

B

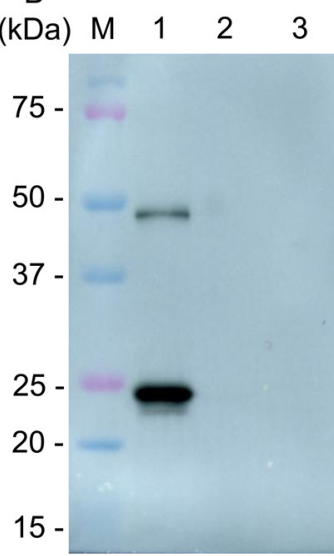

C

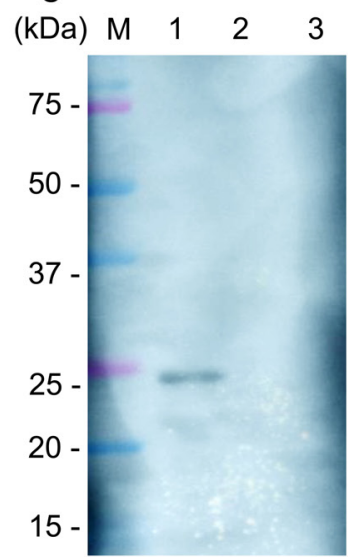

D

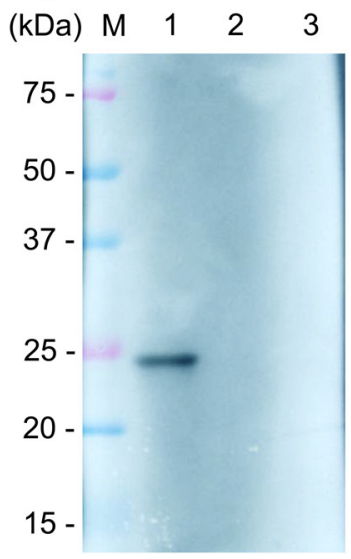

$\mathrm{E}$

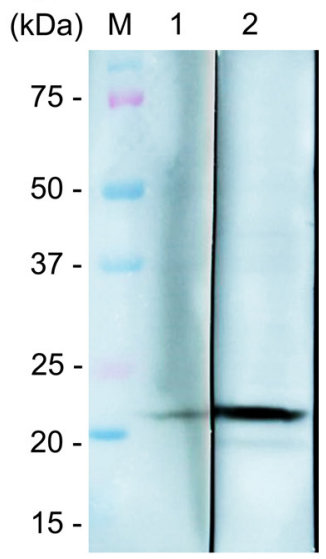

Fig. 2. Western blotting analysis showing the reaction of specific antibodies to antigens. (A to D) Lane M - Molecular weight marker, Lane 1 - His tagged recombinant SjTPx-1, Lane 2 - His tagged recombinant MmPrx-1, Lane 3 - His tagged recombinant HsPrx-1. (E) Lane M - Molecular weight marker, Lane 1 and Lane $2-$ S. japonicum adult worm lysate. (A) shows the positive reactions of all His tagged rSjTPx-1, rMmPrx-1 and rHsPrx-1 to anti 6x-his tag antibody. (B to D) shows the specific reaction of mAbA1F7, A1F11 and A1G7 to SjTPx-1 but not to the mammalian orthologue Prx-1 respectively. (E) shows the recognition of the native SjTPx-1 as $22 \mathrm{kDa}$ polypeptide by both anti SjTPx-1 mAb (mAbA1F7; lane 1) and pAb (lane 2) from adult worm lysate.
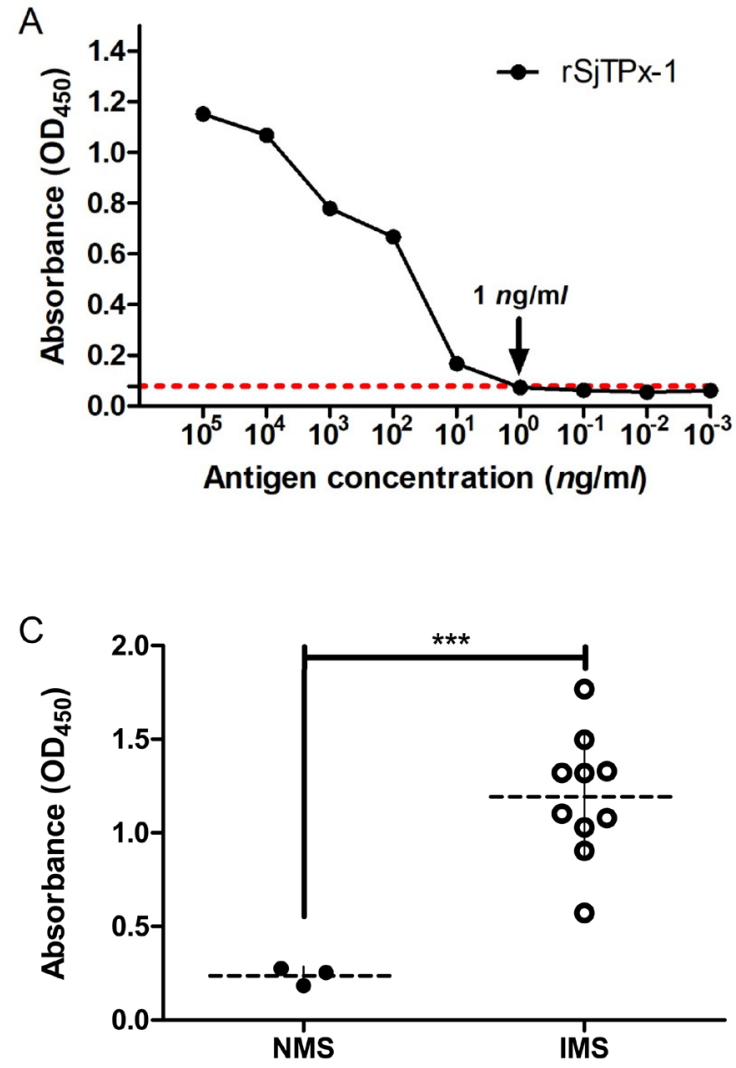

B

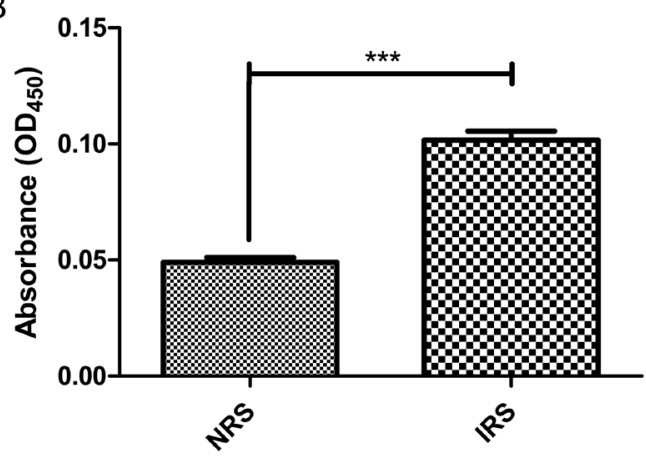

D

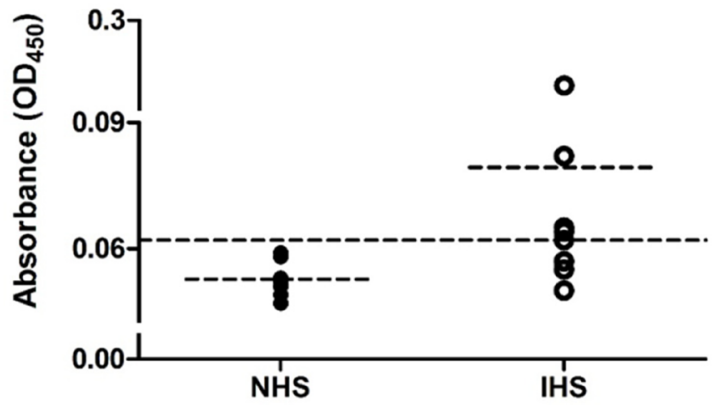

Fig. 3. DAS-ELISA optimization and performance. (A) shows the least amount of detectable antigen concentration detected by the system. Broken line shows the cut-off point and the dots signify $\mathrm{OD}_{450}$ reading of the recombinant $\mathrm{SjTPx}-1$ at each dilution factor. The detection limit is determined to be $1 \mathrm{ng} / \mathrm{ml}$. (B) shows the testing of normal rabbit serum (NRS) and experimentally infected rabbit serum (IRS). Error bars represent standard deviation (SD). Asterisks show the significant difference $(P \leq 0.001)$ in mean $\mathrm{OD}_{450}$ of triplicate readings between NRS and IRS. (C) show the testing of normal mouse sera $(\mathrm{n}=3)(\mathrm{NMS})$ and experimentally infected mouse sera $(\mathrm{n}=10)$ (IMS). The short dotted lines at each group shows the mean absorbance reading between normal and infected mouse sera. Asterisks showed significant difference $(P \leq 0.001)$ in mean $\mathrm{OD}_{450}$ of triplicate readings between NMS and IMS. (D) shows the testing of human clinical cases ( $\left.\mathrm{n}=10\right)$. Dotted line across the graph signifies the cut-off point. The short dotted line at each group shows the mean absorbance reading for normal (NHS) and infected human sera (IHS). This graph shows that 4 out of the 10 samples were shown to have detectable antigen levels. 
detecting excretory and secretory antigens is beneficial for indicating active infection, effect of chemotherapy and disease severity [12]. Here, we used serum as the biological sample for the detection of the target antigen. Although the current available antigenbased POCT for schistosomiasis diagnosis uses urine as sample. Previous studies on circulating antigens has shown that using serum samples for antigen detection gave superior specificity as compared to using urine as samples [2, 26, 35]. This might be attributed to the fact that since adult worms inhabit the mesenteric veins any secreted product derived from the worm are carried through the blood circulation first, prior to other bodily fluids including urine. It should also be noted that as compared to CCA, the target antigen (SjTPx-1) has not yet been proven to be present in the urine of the infected host which leaves an avenue for further studies.

SjTPx-1, a component of a repertoire of proteins excreted and secreted by the adult worm was cloned and expressed as a histidine tagged fusion protein. This recombinant antigen was used to produce specific antibodies; a rabbit pAb as capture antibody and a mouse mAb as detection antibody in DAS-ELISA format. Because of the ubiquitous nature of TPx-1, the mAb used as detection antibody was verified by Western blotting for specificity to parasite TPx-1 and not react to the mammalian orthologues represented by recombinant mouse Prx-1 and human Prx-1. Furthermore, these pAb and mAb were shown to recognize native $\mathrm{SjTPx}-1$ derived from the adult worm indicating that the recombinant $\mathrm{SjTPx}-1$ is antigenically similar to the native protein. Taken together, these results suggest that both $\mathrm{pAb}$ and $\mathrm{mAb}$ produced against $\mathrm{rSjTPx}-1$ were suitable capture and detection antibodies for DAS-ELISA. The optimal concentrations of capture pAb and detection mAb were determined by checkerboard titration at 8 and 1 $\mu \mathrm{g} / \mathrm{ml}$, respectively. Serial dilution of the recombinant antigen showed that at least $1 \mathrm{ng} / \mathrm{m} l$ of the antigen can be detected by this method. The detection limit of this method is much lower than CCA at $5 \mathrm{ng} / \mathrm{ml}$ and comparable to the detection limit established for circulating anodic antigen (CAA) at $1.1 \mathrm{ng} / \mathrm{m} l$ that have been used for the diagnosis of $S$. mansoni and S. haematobium infection but not for $S$. japonicum infection $[9,23]$. Several circulating antigens with detection capabilities comparable to ours or even higher have also been found in previous studies as potential antigens for diagnosis of Asian schistosomiasis [10, 11, 17, 22].

The DAS-ELISA was able to demonstrate the presence of the target antigen in all the infected rabbit and mouse sera, indicating that SjTPx-1 could be used as a biomarker for schistosomiasis diagnosis in experimentally infected animals. In order to determine the diagnostic potential of SjTPx-1 in human clinical samples. 10 stool-confirmed human serum samples were evaluated; 4 of which had detectable levels of the target antigen in the serum. This result suggests that the target antigen is present and detectable in the patient serum. The stage of infection when this antigen can readily be detected in the serum should be investigated, since it was known in other antigen detection methods that inherent factors including fluctuation in the antigen secretion may affect the target antigen detectability of the test [27]. All serum samples tested were determined positive by indirect ELISA with rSjTPx-1 as antigen (data not shown). However, there was no correlation found between the circulating antigen level and antibody level in those serum samples (data not shown). The correlation between the antigen level and the antibody level which may be useful in evaluating status of the infection should also be investigated in the future studies.

As the schistosomiasis endemic countries in Asia including China, the Philippines and Indonesia promote the elimination of this disease $[25,31,36]$, the WHO further intensified its commitment in eliminating this neglected tropical disease by targeting a global coverage of at least $75 \%$ of preventive chemotherapy for schistosomiasis in 2020 . Currently, the only available antigenbased serological diagnosis assay for schistosomiasis is the POCT based on Schistosoma mansoni CCA; this has been shown to have suboptimal sensitivity when applied to $S$. japonicum infection [33, 34]. The continuous use of the laborious and low sensitive microscopy-based diagnostic test may potentially interrupt the efforts put into the elimination of this disease. The development of a test that can identify the true status of infection based on parasite derived molecules will be very useful in the effective screening and evaluation of the control measures being implemented for Asian zoonotic schistosomiasis. Determination of active infection by detecting circulating cell-free DNA of the parasite in serum and urine samples from the patients have also been reported [14]. However, the detection of the DNA is PCR based, and it requires an expensive machine which may not be affordable in rural clinical settings [37]. On the other hand, the detection of circulating antigen which can be applied with POCT may have an advantage if its introduction to the field and rural clinical settings are considered.

In this study, we evaluated and confirmed that SjTPX-1 could be detected in the serum of experimentally infected animals and human clinical cases. However, additional clarification in relation to the antigen dynamics shall be done such as: identification of peak secretion post-infection, response of antigenemia after treatment as well as testing of more clinical samples in order to determine applicability in field setting. Application of the test for urine sample as well as serum sample should also be addressed in the future study. Results of this study paves the way for the potential use of SjTPx-1 as a candidate circulating antigen that can be applied to diagnose schistosomiasis japonica infection.

ACKNOWLEDGMENTS. We thank Dr. Keisuke Suganuma and Dr. Shino Yamasaki from the National Research Center for Protozoan Diseases, Obihiro University of Agriculture and Veterinary Medicine (NRCPD/OUAVM) for their suggestions and technical assistance in the monoclonal antibody development. We thank Ms. Ntathisi Innocentia Molefe of NRCPD/OUAVM for providing biological samples for production of recombinant MmPrx-1.

\section{REFERENCES}

1. Alarcón de Noya, B., Ruiz, R., Losada, S., Colmenares, C., Contreras, R., Cesari, I. M. and Noya, O. 2007. Detection of schistosomiasis cases in low-transmission areas based on coprologic and serologic criteria The Venezuelan experience. Acta Trop. 103: 41-49. [Medline] [CrossRef]

2. Al-Sherbiny, M. M., Osman, A. M., Hancock, K., Deelder, A. M. and Tsang, V. C. 1999. Application of immunodiagnostic assays: detection of 
antibodies and circulating antigens in human schistosomiasis and correlation with clinical findings. Am. J. Trop. Med. Hyg. 60: 960-966. [Medline] [CrossRef]

3. Angeles, J. M. M., Goto, Y., Kirinoki, M., Asada, M., Leonardo, L. R., Rivera, P. T., Villacorte, E. A., Inoue, N., Chigusa, Y. and Kawazu, S. 2012. Utilization of ELISA using thioredoxin peroxidase-1 and tandem repeat proteins for diagnosis of Schistosoma japonicum infection among water buffaloes. PLoS Negl. Trop. Dis. 6: e1800. [Medline] [CrossRef]

4. Angeles, J. M. M., Goto, Y., Kirinoki, M., Leonardo, L., Tongol-Rivera, P., Villacorte, E., Inoue, N., Chigusa, Y. and Kawazu, S. 2011. Human antibody response to thioredoxin peroxidase-1 and tandem repeat proteins as immunodiagnostic antigen candidates for Schistosoma japonicum infection. Am. J. Trop. Med. Hyg. 85: 674-679. [Medline] [CrossRef]

5. Angeles, J. M. M., Kirinoki, M., Goto, Y., Asada, M., Hakimi, H., Leonardo, L. R., Tongol-Rivera, P., Villacorte, E. A., Inoue, N., Chigusa, Y. and Kawazu, S. 2013. Localization and expression profiling of a 31 kDa antigenic repetitive protein Sjp_0110390 in Schistosoma japonicum life stages. Mol. Biochem. Parasitol. 187: 98-102. [Medline] [CrossRef]

6. Cesari, I. M., Pujol, F. H., Rodríguez, M. and Alarcón de Noya, B. 1987. Antigenic enzymes of Schistosoma mansoni: possible use for immunodiagnosis. Mem. Inst. Oswaldo Cruz 82 Suppl 4: 175-177. [Medline] [CrossRef]

7. Davis, J. M., Pennington, J. E., Kubler, A. M. and Conscience, J. F. 1982. A simple, single-step technique for selecting and cloning hybridomas for the production of monoclonal antibodies. J. Immunol. Methods 50: 161-171. [Medline] [CrossRef]

8. De Jonge, N., Fillié, Y. E. and Deelder, A. M. 1987. A simple and rapid treatment (trichloroacetic acid precipitation) of serum samples to prevent non-specific reactions in the immunoassay of a proteoglycan. J. Immunol. Methods 99: 195-197. [Medline] [CrossRef]

9. Fillié, Y. E., Van Lieshout, L., Kornelis, D. and Deelder, A. M. 1994. Evaluation of an ELISA for combined measurement of CAA and CCA in schistosomiasis mansoni. Acta Trop. 57: 279-287. [Medline] [CrossRef]

10. Fu, C. and Carter, C. E. 1990. Detection of a circulating antigen in human schistosomiasis japonica using a monoclonal antibody. Am. J. Trop. Med. Hyg. 42: 347-351. [Medline] [CrossRef]

11. Gao, H., Xiao, D., Song, L., Zhang, W., Shen, S., Yin, X., Wang, J., Ke, X., Yu, C. and Zhang, J. 2016. Assessment of the diagnostic efficacy of enolase as an indication of active infection of Schistosoma japonicum. Parasitol. Res. 115: 151-164. [Medline] [CrossRef]

12. Gomes, L. I., Enk, M. J. and Rabello, A. 2014. Diagnosing schistosomiasis: where are we? Rev. Soc. Bras. Med. Trop. 47: 3-11. [Medline] [CrossRef]

13. Hakimi, H., Goto, Y., Suganuma, K., Angeles, J. M. M., Kawai, S., Inoue, N. and Kawazu, S. 2015. Development of monoclonal antibodies against Plasmodium falciparum thioredoxin peroxidase 1 and its possible application for malaria diagnosis. Exp. Parasitol. 154: 62-66. [Medline] [CrossRef]

14. Kato-Hayashi, N., Leonardo, L. R., Arevalo, N. L., Tagum, M. N. B., Apin, J., Agsolid, L. M., Chua, J. C., Villacorte, E. A., Kirinoki, M., Kikuchi, M., Ohmae, H., Haruki, K. and Chigusa, Y. 2015. Detection of active schistosome infection by cell-free circulating DNA of Schistosoma japonicum in highly endemic areas in Sorsogon Province, the Philippines. Acta Trop. 141 Pt B: 178-183. [Medline] [CrossRef]

15. Kumagai, T., Osada, Y. and Kanazawa, T. 2006. 2-Cys peroxiredoxins from Schistosoma japonicum: the expression profile and localization in the life cycle. Mol. Biochem. Parasitol. 149: 135-143. [Medline] [CrossRef]

16. Lee, T. H., Yu, S. L., Kim, S. U., Lee, K. K., Rhee, S. G. and Yu, D. Y. 1999. Characterization of mouse peroxiredoxin I genomic DNA and its expression. Gene 239: 243-250. [Medline] [CrossRef]

17. Lei, J. H., Liu, W. Q., Sun, C. S., Tang, C. L., Li, M. J., Chen, Y. L. and Li, Y. L. 2009. Detection of circulating antigen in serum of mice infected with Schistosoma japonicum by immunomagnetic bead ELISA based on IgY. Acta Trop. 111: 39-43. [Medline] [CrossRef]

18. Leonardo, L. R., Acosta, L. P., Olveda, R. M. and Aligui, G. D. L. 2002. Difficulties and strategies in the control of schistosomiasis in the Philippines. Acta Trop. 82: 295-299. [Medline] [CrossRef]

19. Lier, T., Simonsen, G. S., Wang, T., Lu, D., Haukland, H. H., Vennervald, B. J. and Johansen, M. V. 2009. Low sensitivity of the formol-ethyl acetate sedimentation concentration technique in low-intensity Schistosoma japonicum infections. PLoS Negl. Trop. Dis. 3: e386. [Medline] [CrossRef]

20. Liu, F., Cui, S. J., Hu, W., Feng, Z., Wang, Z. Q. and Han, Z. G. 2009. Excretory/secretory proteome of the adult developmental stage of human blood fluke, Schistosoma japonicum. Mol. Cell. Proteomics 8: 1236-1251. [Medline] [CrossRef]

21. Mulvenna, J., Moertel, L., Jones, M. K., Nawaratna, S., Lovas, E. M., Gobert, G. N., Colgrave, M., Jones, A., Loukas, A. and McManus, D. P. 2010a. Exposed proteins of the Schistosoma japonicum tegument. Int. J. Parasitol. 40: 543-554. [Medline] [CrossRef]

22. Nibbeling, H. A. M., Kahama, A. I., Van Zeyl, R. J. M. and Deelder, A. M. 1998. Use of monoclonal antibodies prepared against Schistosoma mansoni hatching fluid antigens for demonstration of Schistosoma haematobium circulating egg antigens in urine. Am. J. Trop. Med. Hyg. 58: 543-550. [Medline] [CrossRef]

23. Nogueira-Queiroz, J. A., Lutsch, C., Capron, M., Dessaint, J. P. and Capron, A. 1986. Detection and quantification of circulating antigen in schistosomiasis by a monoclonal antibody. I. Specificity analysis of a monoclonal antibody with immunodiagnostic capacity. Clin. Exp. Immunol. 65: 223-231. [Medline]

24. Ochodo, E. A., Gopalakrishna, G., Spek, B., Reitsma, J. B., van Lieshout, L., Polman, K., Lamberton, P., Bossuyt, P. M. M. and Leeflang, M. M. G. 2015. Circulating antigen tests and urine reagent strips for diagnosis of active schistosomiasis in endemic areas. Cochrane Database Syst. Rev. $\mathbf{3}$ : CD009579. [Medline]

25. Olveda, D. U., Li, Y., Olveda, R. M., Lam, A. K., McManus, D. P., Chau, T. N. P., Harn, D. A., Williams, G. M., Gray, D. J. and Ross, A. G. P. 2014. Bilharzia in the Philippines: past, present, and future. Int. J. Infect. Dis. 18: 52-56. [Medline] [CrossRef]

26. Polman, K., Stelma, F. F., Gryseels, B., Van Dam, G. J., Talla, I., Niang, M., Van Lieshout, L. and Deelder, A. M. 1995. Epidemiologic application of circulating antigen detection in a recent Schistosoma mansoni focus in northern Senegal. Am. J. Trop. Med. Hyg. 53: 152-157. [Medline] [CrossRef]

27. Polman, K., Engels, D., Fathers, L., Deelder, A. M. and Gryseels, B. 1998. Day-to-day fluctuation of schistosome circulating antigen levels in serum and urine of humans infected with Schistosoma mansoni in Burundi. Am. J. Trop. Med. Hyg. 59: 150-154. [Medline] [CrossRef]

28. Ren, C. P., Liu, Q., Liu, F. C., Zhu, F. Y., Cui, S. X., Liu, Z., Gao, W. D., Liu, M., Ji, Y. S. and Shen, J. J. 2017. Development of monoclonal antibodies against $\mathrm{Sj} 29$ and its possible application for schistosomiasis diagnosis. Int. J. Infect. Dis. 61: 74-78. [Medline] [CrossRef]

29. Ross, A. G., Olveda, R. M., Acosta, L., Harn, D. A., Chy, D., Li, Y., Gray, D. J., Gordon, C. A., McManus, D. P. and Williams, G. M. 2013. Road to the elimination of schistosomiasis from Asia: the journey is far from over. Microbes Infect. 15: 858-865. [Medline] [CrossRef]

30. Sangfuang, M., Chusongsang, Y., Limpanont, Y., Vanichviriyakit, R., Chotwiwatthanakun, C., Sobhon, P. and Preyavichyapugdee, N. 2016. Schistosoma mekongi cathepsin B and its use in the development of an immunodiagnosis. Acta Trop. 155: 11-19. [Medline] [CrossRef]

31. Satrija, F., Ridwan, Y., Jastal., Samarang and Rauf, A. 2015. Current status of schistosomiasis in Indonesia. Acta Trop. 141 Pt B: $349-353$. [Medline] [CrossRef] 
32. Sulbarán, G. S., Ballen, D. E., Bermúdez, H., Lorenzo, M., Noya, O. and Cesari, I. M. 2010. Detection of the Sm31 antigen in sera of Schistosoma mansoni- infected patients from a low endemic area. Parasite Immunol. 32: 20-28. [Medline] [CrossRef]

33. van Dam, G. J., Wichers, J. H., Ferreira, T. M. F., Ghati, D., van Amerongen, A. and Deelder, A. M. 2004. Diagnosis of schistosomiasis by reagent strip test for detection of circulating cathodic antigen. J. Clin. Microbiol. 42: 5458-5461. [Medline] [CrossRef]

34. van Dam, G. J., Odermatt, P., Acosta, L., Bergquist, R., de Dood, C. J., Kornelis, D., Muth, S., Utzinger, J. and Corstjens, P. L. 2015. Evaluation of banked urine samples for the detection of circulating anodic and cathodic antigens in Schistosoma mekongi and S. japonicum infections: a proof-ofconcept study. Acta Trop. 141 Pt B: 198-203. [Medline] [CrossRef]

35. Van Lieshout, L., Panday, U. G., De Jonge, N., Krijger, F. W., Oostburg, B. F., Polderman, A. M. and Deelder, A. M. 1995. Immunodiagnosis of schistosomiasis mansoni in a low endemic area in Surinam by determination of the circulating antigens CAA and CCA. Acta Trop. 59: 19-29. [Medline] [CrossRef]

36. Wang, J. L., Li, T. T., Huang, S. Y., Cong, W. and Zhu, X. Q. 2016. Major parasitic diseases of poverty in mainland China: perspectives for better control. Infect. Dis. Poverty 5: 67. [Medline] [CrossRef]

37. Weerakoon, K. G. A. D., Gobert, G. N., Cai, P. and McManus, D. P. 2015. Advances in the diagnosis of human schistosomiasis. Clin. Microbiol. Rev. 28: 939-967. [Medline] [CrossRef]

38. WHO. (2017 January updated). Schistosomiasis fact sheet. Retrieved from http://www.who.int/mediacentre/factsheets/fs $115 /$ en/ (accessed February 9, 2017). 